\title{
An AAL3/4-based Architecture for Interconnection between ATM and Cellular Networks
}

\author{
S.M. Jiang, Danny H.K. Tsang, Samuel T. Chanson \\ Hong Kong University of Science \& Technology \\ Clear Water Bay, Kowloon, Hong Kong
}

ABSTRACT: With the increased popularity of ATM technology and the high demand for multimedia applications in wireless systems, the interconnection between wireless systems and wired ATM networks becomes important to support multimedia applications. This paper presents an AAL3/4-based architecture for the interconnection between wireless and ATM networks. The main advantage of this architecture is that modification or additional functions are not necessary to the ATM layer to support mobility, and the ATM part of the connection is totally transparent to the mobiles so that the existing wireless protocols can be used without any change.

\section{INTRODUCTION}

Recent research on wireless systems suggests the use of high-speed wired networks as backbone to support multimedia applications in mobile networks. Since Asynchronous Transfer Mode (ATM) has been accepted as the standard for future high-speed networks, the interconnection between wireless systems and wired ATM networks becomes an important issue. An important design issue of mobile cellular systems is handoff support since handoff happens frequently in micro/pico cellular systems. When handoff occurs, the involved connection needs to be reset to maintain the promised QoS. If a connection has to be reset for each handoff in the micro/pico cellular system, the network may be overloaded by the signaling traffic for resetting connections.

Some suggestions to alleviate this problem have been discussed in the literature. An effort to avoid the entire connection to be affected by handoffs is to split the end-to-end connection into a relatively static portion and a dynamic portion which connects with the mobile, such as virtual connection tree (VCT) [4], mobile representative [9] and bridging point [8]. When handoff occurs, the dynamic portion is reset first, and then the static portion is reset if necessary. This approach requires the ATM layer to have the capability of partially resetting an ATM connection, which includes the signaling protocols for splitting a connection and joining two connections into one. Unfortunately, such procedures do not exist in the current standards of the ATM layer.

Another effort to avoid the entire ATM connection from being affected by handoffs is to allow the mobiles which are present in the same source cell and are roaming to the same destination cell to share a common connection. One way to do this at the ATM layer is using pre-established virtual paths (VPs) to link the adjacent base stations, and using virtual connections (VCs) for handoffs occurring between adjacent base stations. With this approach, a VC is dedicated to a particular mobile. When the mobile roams to a new cell, the corresponding $\mathrm{VC}$ needs to be reset so that the resetting procedure needs to be quick enough to avoid QoS degradation during handoff. To this end, one can adopt the path extension approach [8], i.e., extending a VC from the current cell to the new one. However, a special function at the ATM layer for extending VCs among base stations needs to be added to the current ATM signaling protocol. Other approaches at higher layers such as IP-over-ATM which cannot provide QoS guarantee are not discussed here.

It is definitely necessary to enhance the current ATM definitions to support mobility in broadband wireless networks [2][3][5][7][8]. However, we cannot ignore the fact that there already exist many ATM products following the current ATM definitions, and also many wireless systems such as mobile telephone networks which are not compatible with ATM. Therefore, we proposed an ATM Adaptation Layer (AAL) service type 3/4 (AAL3/4) based architecture for interconnection between wireless cellular systems and wired ATM networks. This architecture requires no change to the existing wireless systems and allows the ATM networks to support mobility without modifications to the ATM layer.

The remainder of the paper is organized as follows. In Section II, we explain the reason for adopting AAL3/4 for the proposed interconnection architecture. In Section III detailed descriptions of the proposal is given, and Section IV concludes the paper.

\section{Motivation for using AAL3/4}

In wireless systems connected by an ATM backbone, an end-to-end connection can be divided into two segments: the wired ATM and the wireless channel. Since the wired ATM network promises to provide QoS guarantees, the endto-end QoS guarantee mainly depends on the QoS provided by the wireless channel. Air space is a shared-medium where a medium access control (MAC) protocol needs to be implemented to ensure equal access opportunity to the mobiles. Obviously, the MAC protocol plays an important role in the QoS provided by the wireless channel. The access delay introduced by the MAC layer (the interval from a packet arrives at the MAC layer until it is transmitted) will affect the end-to-end delay of the connection. Therefore, the QoS guarantee provided by the air media depends heavily on the MAC protocol. Detailed discussion on MAC is beyond the scope of the paper. In the following, we discuss only handoff support with the existing ATM products. In our view, this problem should be considered at the ATM Adaptation Layer (AAL) or the layers above AAL because the ATM layer of the existing products cannot be modified.

There are four types of AAL services proposed by ITU-T, 
i.e., AAL1, AAL2, AAL3/4 and AAL5. AAL1 was originally designed for constant bit rate applications such as voice, AAL2 for variable bit rate and delay-sensitive applications such as video, and AAL3/4 as well as AAL5 for data applications. Despite the service-specific functions provided by the AAL layers, the QoS of the AAL layer will eventually be determined by the QoS of the ATM layer. In other words, AAL3/4 and AAL5 may also be able to support delaysensitive applications as long as the corresponding ATM connections can satisfy the required QoS.

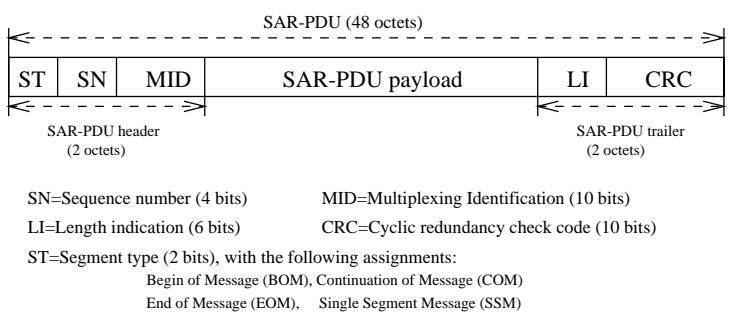

Fig. 1. Format of AAL3/4 SAR-PDU

However, AAL3/4 has some features which are not available in the other AAL services due to its specific structure which is designed to support connectionless applications. As shown in Figure 1, the AAL3/4 SAR-PDU contains a multiplexing identification (MID) and some other fields. These fields provide the multiplexing capability which permits several applications to share a common ATM connection. As shown in Figure 2 [6], the AAL3/4_Service Access Point (SAP) is provided at the AAL interface for the AAL3/4 service access. Each AAL3/4_SAP has its own MID value assigned by the AAL layer. The data from different AAL3/4_SAPs may be interleaved to a common ATM connection at the sender side which will be demultiplexed at the receiver side. Using AAL3/4 for interconnecting cellular systems and wired ATM networks has the following advantages:

1) With the AAL3/4 multiplexing capability, a preestablished ATM connection between a pair of adjacent radio cells can be shared by several mobiles. This paper assumes handoffs occurs only at cell boundaries. This is useful in dealing with frequent handoffs since multiplexing avoids the necessity of establishing an individual ATM connection for each handoff.

2) The MID of AAL3/4 can be used as the routing tag at the AAL layer. A type of flexible connection to link any cells can be formed by MID switching at the AAL layer based on the pre-established ATM connections between adjacent cells. Alternatively, flexible connections can be formed by using AAL3/4_SAP switching at the AAL interface. The details of these two types of connections are given in Section III.

3) The AAL3/4 SAR-PDU can be used to provide payloadlevel multiplexing, i.e., allowing data from different applications to share an ATM cell [1], without modifications to its format structure.

\section{The PRoposed architecture}

As shown in Figure 3, the proposed architecture allows the air medium design independent of the wireline design. A

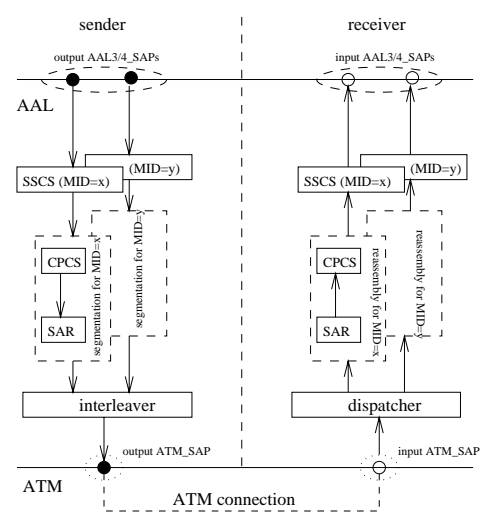

Fig. 2. Functional model for AAL3/4 type

pre-established ATM connection is maintained between every pair of adjacent base stations. These connections may be permanent, semi-permanent or switched. It is possible to use ATM connections to link non-adjacent base stations directly. However, to support frequent handoffs in the micro/pico cellular system without causing a large volume of signaling traffic to the ATM network, AAL3/4 based connections over the ATM layer can be employed, which can extend between any base stations by using the pre-established ATM connections between adjacent base stations. Therefore, the AAL3/4 based connections can be extended with the movement of mobiles for handoffs. On the other hand, the proposed architecture can provide higher granularity multiplexing capability by using the AAL3/4 SAR-PDU, i.e., allowing data from different applications to share an ATM cell [1]. This type of multiplexing not only satisfies delay-stringent applications, but also improves the utilization of the ATM cell payload. In the following, we describe in details the structure of the proposed architecture.

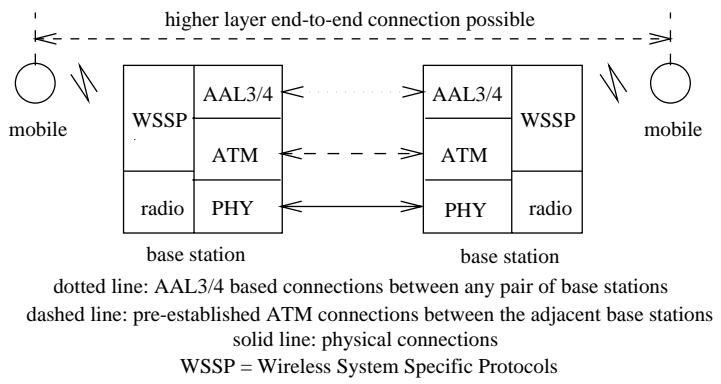

Fig. 3. Protocol stacks for interconnection between base stations

\section{A. AAL3/4 based connections}

As shown in Figure 2, the AAL3/4_SAP is provided at the AAL interface for accessing the AAL3/4 service. Each AAL3/4_SAP corresponds to a unique MID value within an ATM connection at the AAL layer. (Note that the same MID value may be assigned to different AAL connections interleaved in different ATM connections.) Therefore, the AAL3/4 based connections are supported by AAL3/4 multiplexing with MID in the pre-established ATM connections between adjacent cells as shown in Figure 4. This figure 


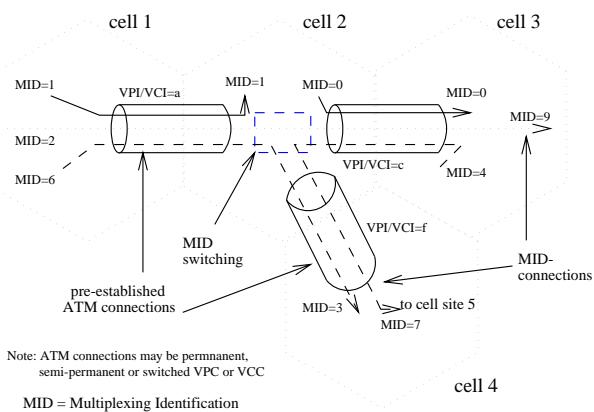

Fig. 4. Pre-established ATM connections and AAL3/4-based connections

TABLE I

INPUT AAL3/4_SAP LOOKUP-TABLE

\begin{tabular}{||c|c||}
\hline Input AAL3/4_SAP & Output of incoming data \\
\hline \hline SAP 3 & here \\
\hline SAP 5 & SAP 4 \\
\hline
\end{tabular}

[Note]: Table content for cell 2 in Figure 5

shows also an example of one type of AAL3/4 based connection which is formed by switching MID, called MIDconnection. Another type of AAL3/4 based connection is formed by linking the AAL3/4_SAPs at the AAL interface which is referred to as SAP-connection for the discussion purpose in this paper.

\section{A.1 SAP-connection at the AAL interface}

At the AAL interface, a user can only see the AAL3/4_SAP since everything below the interface is transparent to it. However, as shown in Figure 2, an AAL3/4_SAP is the access point to a particular cell (different from ATM cell). Therefore, switching AAL3/4_SAP at the AAL interface can extend a connection to any cells. To this end, two tables are necessary for such switching. Table I maintains information about the destination or the output AAL3/4_SAP for the incoming data from the input AAL3/4_SAPs. Table II stores the information on the output AAL3/4_SAPs corresponding to the destination cells.

Figure 5 gives an example of the SAP-connection. As shown in Table I, the output of the data from an input AAL3/4_SAP can be found in "Output of incoming data" which may be set to either 'here' or the 'AAL3/4_SAP' corresponding to some other cell. 'here' means the destination of the incoming data is the current cell, and the data will be forwarded to higher layers for further processing. With this table, an intermediate cell can forward the data from

TABLE II

OUTPUT AAL3/4_SAP LOOKUP-TABLE

\begin{tabular}{||c|c||}
\hline Destination cell & Output AAL3/4_SAP \\
\hline \hline cell 3 & SAP 4 \\
\hline cell 4 & SAP 2 \\
\hline
\end{tabular}

[Note]: Table content for cell 2 in Figure 5 one cell to another by linking the input AAL3/4_SAP to the proper output AAL3/4_SAP (e.g., between SAPs 5 and 4 in cell 2 in Figure 5) to extend the original connection. The output AAL3/4_SAP corresponding to the destination cell (e.g., SAP to cell 3 from cell 2 in Figure 5) can be found in Table II. This table may also include other information such as the QoS an AAL3/4_SAP supports.

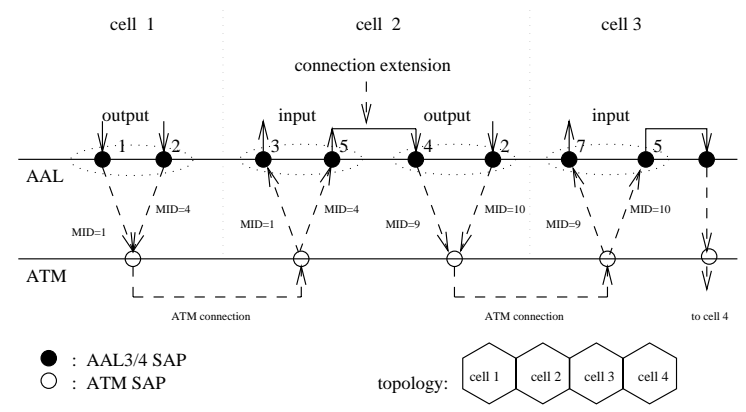

Fig. 5. Example of SAP-connections at the AAL interface

\section{A.2 MID-connection at the AAL layer}

The MID-connection is formed by using the MID as the routing tag at the AAL layer as shown in Figure 4. If several mobiles are moving to the same cell from the same source cell, they can share a common pre-established ATM connection with different MIDs. The AAL layer at an intermediate cell (e.g., cell 2 in Figure 4) checks the MID values of all incoming SAR-PDUs. If the destination of an incoming SAR-PDU is the current cell, then the SAR-PDU is passed to the SAR sublayer for further processing; otherwise, the SAR-PDU is assigned a new MID and forwarded to another cell via the proper ATM_SAP. Figure 6 shows the switching diagram for the MID-connections given in Figure 4.

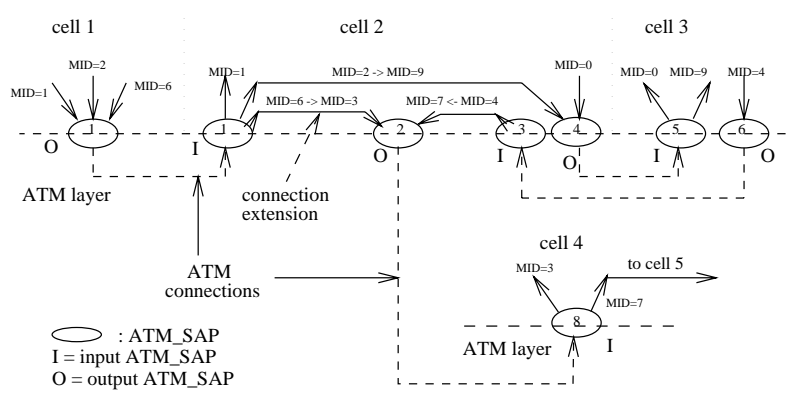

Fig. 6. Switching diagram for MID-connections given in Figure 4

Similar to the SAP-connection at the AAL interface, there are also two tables for the MID-connections at the AAL layer. Table III maintains the information on the output of the incoming SAR-PDUs. If the destination of an incoming SARPDU is the current cell, then the "Here/Other" field is set to 'here'; otherwise, the ATM_SAP corresponding to the next cell and new MID value for the SAR_PDU are given. Table IV provides information on the output ATM_SAPs corresponding to the destination cells. Other information such as the QoS provided by an ATM_SAP can also be put in this table. 
TABLE III

INPUT ATM_SAP AND MID LOOKUP-TABLE

\begin{tabular}{||c|c|c|c||}
\hline \multicolumn{2}{|c|}{ Input } & \multicolumn{2}{c||}{ Output of incoming data } \\
\hline ATM_SAP & MID & Here/Other & New MID \\
\hline \hline SAP 1 & 1 & here & empty \\
\hline SAP 1 & 2 & SAP 4 & 9 \\
\hline SAP 1 & 6 & SAP 2 & 3 \\
\hline SAP 3 & 4 & SAP 2 & 7 \\
\hline
\end{tabular}

TABLE IV

OUTPUT ATM_SAP AND MID LOOKUP-TABLE

\begin{tabular}{||c|c|c||}
\hline Output ATM_SAP & MID & Destination cell \\
\hline \hline SAP 2 & 3 & cell 4 \\
\hline SAP 2 & 7 & cell 5 \\
\hline SAP 4 & 0 & cell 3 \\
\hline SAP 4 & 9 & cell 3 \\
\hline \multicolumn{2}{|c|}{ [Note]: Table content for cell 2 in Figure 6}
\end{tabular}

[Note]: Table content for cell 2 in Figure 6

A.3 Comparison between SAP-connection

and

MID-connection

The main advantage of the SAP-connection at the AAL interface is that modifications or addition functions are not necessary at both the ATM layer and the AAL layer. However, an extra delay is caused by the reassembly of the incoming SAR-PDUs into an AAL-Service Data Unit (SDU) if the destination of the AAL-SDU is not the current cell. In this case, the AAL-SDU will return to the AAL layer again for segmentation. This extra delay is avoided by the MID-connection at the expense of modifications to the AAL layer. MID switching for the MID-connection can be done at the dispatcher of the receiver side (see Figure 2) without reassembling of incoming SAR-PDUs into AAL-SDUs.

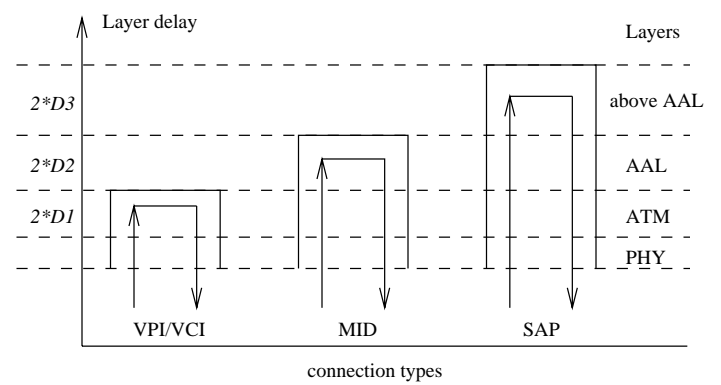

Fig. 7. Diagram of layer-delays versus connection types

On the other hand, the delays suffered by a data unit over the AAL layer for the SAP-connection and at the AAL layer for the MID-connection are different as shown in Figure 7. These layer-delays consist of two portions corresponding to data movements between layers: up and down. We suppose the up and down delays of a particular layer are the same for simplicity. Denote $D 1$ as the layer-delay at the ATM layer in one direction, $D 2$ at the AAL layer and $D 3$ above the AAL layer. $D 1 \leq D 2 \leq D 3$ since the switching at the ATM layer is done by hardware and those at the AAL layer or above may be partially or completely done by the software. Therefore, the ATM connection is the most efficient among the three types of connections. However, to avoid modifications to the ATM layer, the MID-connection is a good choice. If the applications such as data can tolerate the delay caused by the SAP-connection, it should be the most economical choice since no modification is needed to both the ATM and AAL layers.

\section{B. AAL3/4 for Payload-level multiplexing (PLM)}

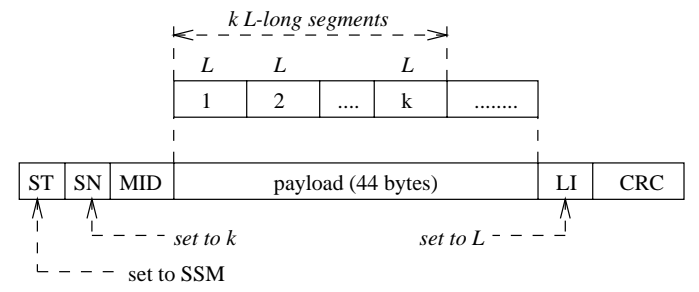

Fig. 8. AAL3/4 SAR-PDU format for PLM

Some delay-stringent applications such as voice may still be sensitive to the delay caused by waiting to accumulate enough information to pack the payload of an ATM cell. For example, it takes about $20 \mathrm{~ms}$ with TDMA and CDMA to pack the payload of an ATM cell [1]. Therefore, [1] suggested to multiplex the information from different mobiles into an ATM cell.

\section{B.1 Multiplexing structure}

A simple way to do share an ATM cell is to divide the payload of an ATM cell into multiple equal-length segments. The existing format of the AAL3/4 SAR-PDU [6] can be used directly for such multiplexing without modification. As shown in Figure 1, the ST of SAR-PDU identifies four types of segments for connectionless messages: BOM, COM, EOM and SSM. The first three segment types along with the SN are used for transmission of long messages which have to be fragmented into SN pieces of AAL3/4 payload units (i.e., 44 bytes). However, when the size of a Common Part Convergence Sublayer-Protocol Data Unit (CPCS-PDU) is smaller than or equal to 44 bytes (i.e., ST $=\mathrm{SSM}$, which is always the case with PLM), the SN setting is not necessary for the normal AAL3/4 usage but can be used for PLM. We define the combination ' $\mathrm{ST}=\mathrm{SSM}$ and $\mathrm{SN}=0$ ' indicates 'Single Segment Message' for the normal AAL3/4 usage; and 'ST=SSM and SN $>0$ ' is used for PLM as shown in Table V. Suppose a part or all of the 44-byte payload of an AAL3/4 SAR-PDU is divided into $k$ segments, each of which has the same size $L$ as shown in Figure 8. Thus, $\mathrm{SN}$ is used to denote $k$ and LI denote $L$ for PLM.

The PLM can only be used by the MID-connection since it operates directly on the AAL3/4 SAR-PDUs. The peer communication entities at the AAL layer negotiate in advance the allocation of the segments of the AAL3/4 payload. That is, the source needs to tell the destination which of the $k$ segments of the AAL3/4 payload are allocated to which sessions during connection setup. This may require a map table 
TABLE V

DEFINITION OF SN AND LI FOR NORMAL AAL3/4 AND PLM

\begin{tabular}{||c|c|c|c||}
\hline ST & SN & LI & Definition \\
\hline \hline BOM & $\geq 0$ & $>0$ & normal AAL3/4 \\
\hline COM & $>0$ & $>0$ & normal AAL3/4 \\
\hline EOM & $>0$ & $>0$ & normal AAL3/4 \\
\hline SSM & $=0$ & $>0$ & normal AAL3/4 \\
SSM & $>0$ & $>0$ & PLM \\
\hline
\end{tabular}

for the maintenance between Segment Number and Session Identification at both the source and destination.

\section{B.2 Payload utilization with PLM}

Since the payload of an ATM cell is divided into segments for multiplexing, some segments may be empty for lack of data to pack them during a defined period. This period is the maximum period allowed for packing the entire payload of an ATM cell (packet-time). With PLM, an ATM cell must be transmitted even if there are still empty segments available in the payload when the packet-time is reached. In the following we analyze the payload utilization with PLM for a connection between a pair of adjacent base stations.

Let $m$ be the number of the base stations adjacent to a given base station $B 0$, and $n$ the number of data units received by $B 0$ for transmission to other base stations during a packet-time. Suppose the entire payload of an ATM cell is divided into $k(k>0)$ equal-length segments, and the length of the segment is assumed to be equal to the size of the data unit received by $B 0$. Furthermore, assume $k \leq n$ (in a saturation state) since if $k>n$, at least $(k-n)$ segments in each ATM cell will be empty which will result in low utilization (the maximum utilization in this case is $\frac{n}{k}$ ). Suppose that the destinations of the data from $B 0$ are uniformly distributed among the $m$ adjacent base stations, that is, the probability of the data from $B 0$ going to any one of the $m$ adjacent base stations is $\frac{1}{m}$. Then, the probability that $i$ of the $n$ received data units going to the same base station, $p(i, n)$, is given by:

$$
p(i, n)=\left(\begin{array}{c}
n \\
i
\end{array}\right)\left(\frac{1}{m}\right)^{i}\left(1-\frac{1}{m}\right)^{n-i}, i=0,1, \ldots n
$$

The utilization of the payload of an ATM cell is equal to 1 if all segments of the payload are occupied, that is $i \geq k$; otherwise, the utilization is $\frac{i}{k}$. Therefore, we can have the formula for the mean payload utilization, $\mu(k, n)$, is given by:

$$
\mu(k, n)=\sum_{i=0}^{k-1} p(i, n) \frac{i}{k}+\sum_{i=k}^{n} p(i, n)=1-\sum_{i=0}^{k-1}\left(1-\frac{i}{k}\right) p(i, n)
$$

With Equation (2), we plotted some curves of $\mu(k, n)$ as a function of $n$ by taking $m=6$ and $k=1,2$ and 4 in Figure 9. The horizontal axis is the payload generation rate $(P G R)$. For example, for AAL3/4, $P G R=$ the number of 44-byte

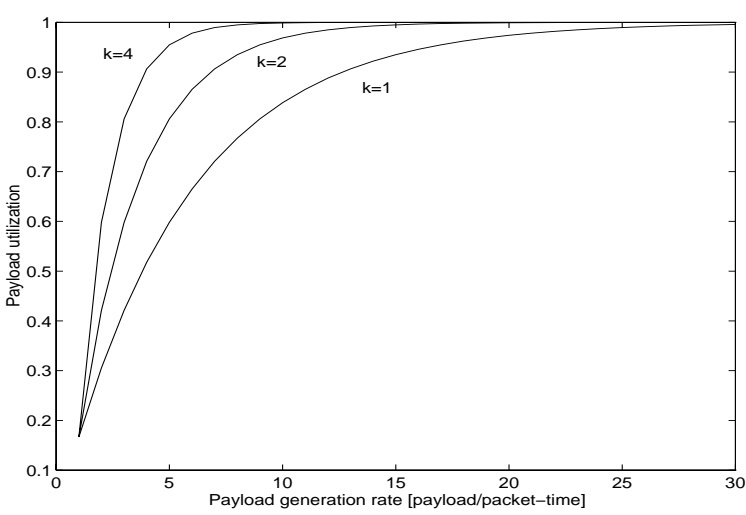

Fig. 9. ATM cell payload utilization versus $n$ for $k \leq n$

payloads received by $B 0$ in a packet-time. Note that $P G R=$ 1 for AAL3/4 indicates one $(n=1)$ 44-byte-payload/packettime for $k=1$, two $(n=2) 22$-byte-payloads/packet-time for $k=2$ and four $(n=4) 11$-byte-payloads/packet-time for $k=4$. As shown in Figure 9, when a payload is divided into larger number of segments for multiplexing, the payload utilization is increased.

\section{Conclusion}

In this paper, an AAL3/4 based architecture has been proposed for interconnection between cellular systems and ATM networks. It provides two types of AAL3/4 based connections for this purpose: SAP-connection and MID-connection. Their common characteristic is that neither modifications nor additional functions are needed to the ATM layer and the existing wireless systems. Furthermore, the SAP-connection mode requires no change even at the AAL layer. A scheme to implement the ATM cell payload level multiplexing based on AAL3/4 has been proposed, which can satisfy delay-stringent applications and improve the ATM cell payload utilization.

\section{REFERENCES}

[1] B. T. Doshi, A. Sawkar, An ATM Based PCS/Cellular Architecture,WINLAB 1995, pp23-35

[2] D. Raychaudhuri, N. D. Wilson, ATM-Based Transport Architecture for Multiservices Personal Communication Networks, IEEE JSAC, Vol. 12, No. 8. October 1994, pp1401-1414

[3] D. Raychaudhuri, L.J. French et al, WATMnet: A Prototype Wireless ATM System For Multimedia Personal Communication, ICC 1996, pp469-477

[4] A. S. Acampora, M. Naghshineh, An Architecture and Methodology for Mobile-Executed Handoff in Cellular ATM Networks, IEEE JSAC, Vol. 12, No. 8. October 1994, pp1365-1375

[5] L. Dellaverson, Wireless ATM Networking and Selected Service Aspects, ATM Forum/95-1569

[6] ITU-T, Recommendation I.363, B-ISDN ATM Adaptation Layer (AAL) Specification

[7] P. Agrawal, E. Hyden et al, SWAN: A Mobile Multimedia Wireless Network, IEEE Personal Communications, April 1996, pp18-33

[8] L. V. Hauwermeiren, L. Vercauteren et al., Requirements For Mobility Support in ATM, GLOBOCOM 1994, pp1691-1695

[9] S. K. Biswas, A. Hopper, A Representative Based Architecture for Handlling Mobility in Connection Oriented Radio Networks, 1995 4th International Conference on Universal Personal Communications, November 6-10, 1995 Japan, pp848-852 